\title{
In-Vivo Functional and Structural Retinal Imaging Using Multiwavelength Photoacoustic Remote Sensing Microscopy and Optical Coherence Tomography
}

\section{Zohreh Hosseinaee}

University of Waterloo

Nicholas Pellegrino

University of Waterloo

Nima Abbasi

University of Waterloo

Tara Amiri

University of Waterloo

James A. Tummon Simmons

University of Waterloo

Paul Fieguth

University of Waterloo

Parsin Haji Reza ( $\nabla$ phajireza@uwaterloo.ca )

University of Waterloo

\section{Research Article}

Keywords: retinal imaging, multiwavelength, photoacoustic remote, microscopy

Posted Date: September 24th, 2021

DOI: https://doi.org/10.21203/rs.3.rs-921966/v1

License: (c) (1) This work is licensed under a Creative Commons Attribution 4.0 International License. Read Full License 
In-vivo functional and structural retinal imaging using multiwavelength photoacoustic remote sensing microscopy and optical coherence tomography

Zohreh Hosseinaee $^{1}$, Nicholas Pellegrino ${ }^{1}$, Nima Abbasi ${ }^{1}$, Tara Amiri ${ }^{1}$, James A. Tummon Simmons ${ }^{1}$,

$$
\text { Paul Fieguth }{ }^{2} \text { Parsin Haji Reza }{ }^{1 *}
$$

1. PhotoMedicine Labs, Department of System Design Engineering, University of Waterloo, 200 University Ave W, Waterloo, ON, N2L 3G1, Canada

2. Department of System Design Engineering, University of Waterloo, 200 University Ave W, Waterloo, ON, N2L 3G1, Canada

*Email: phajireza@uwaterloo.ca 


\section{Abstract.}

A multiwavelength photoacoustic remote sensing (PARS) microscope is combined with sweptsource optical coherence tomography and applied to in-vivo, non-contact retinal imaging. Building on the proven strength of multiwavelength PARS imaging, the system is applied for estimating retinal oxygen saturation in the rat eye. The capability of the technology is demonstrated by imaging both microanatomy and the microvasculature of the retina in-vivo. To our knowledge, this is the first time a non-contact photoacoustic imaging technique is employed for in-vivo oxygen saturation measurement in the retina.

\section{Introduction}

Functional imaging techniques enable measuring alterations in biological function including metabolism, blood flow, regional chemical composition, and biochemical processes. These methods promise to dramatically improve the ability to study in-situ biochemistry and disease pathology ${ }^{1}$. In ophthalmology, functional changes most often precede structural changes in major eye diseases. Detecting these alterations aids in understanding pathogenesis, early diagnosis, and timely management of ophthalmic disorders [2]. Retinal oxygen saturation $\left(\mathrm{SO}_{2}\right)$ and metabolic rate of oxygen consumption $\left(\mathrm{MRO}_{2}\right)$ are among the most important biomarkers characterizing the pathophysiological status of the posterior eye. Additionally, abnormal retinal $\mathrm{SO}_{2}$ levels are believed to be involved in major eye diseases such as diabetic retinopathy (DR) and age-related macular degeneration (AMD) ${ }^{2-4}$. Therefore, the precise measurement of retinal oxygen saturation can be critical in investigating these blinding diseases. Additionally, since the retinal arterioles are derived from the central nervous system. Presuming the retinal arterial oxygen content is identical to the systemic circulation, retinal oximetry may provide relevant information on oxygen delivery to the central nervous system. Therefore, determination of oxygen saturation in the retinal vessels could enhance the monitoring and treatment of critically ill patients in the intensive care units ${ }^{5}$. Oxygen-sensitive electrodes and magnetic resonance imaging have 
been used to measure retinal $\mathrm{SO}_{2}$, however these methods are usually restricted to terminal experiments and/or limited by low spatial resolution ${ }^{3,6}$. Phosphorescence lifetime imaging has been also applied to map ocular oxygenation in animal models. Unfortunately, the need to inject fluorescent probes into systematic circulation makes the method inappropriate for human practice ${ }^{7}$.

Recently, researchers are focused on optical imaging-based methods to evaluate retinal oxygenation. Optical imaging methods are capable of non-invasive $\mathrm{SO}_{2}$ measurement and they can visualize the spatial distribution of oxygenation with high resolution in ocular environment ${ }^{8}$. Optical measurement of $\mathrm{SO}_{2}$ is possible because the two forms of hemoglobin, oxy- and deoxyhemoglobin $\left(\mathrm{HbO}_{2}\right.$ and $\left.\mathrm{Hb}\right)$, have distinct optical absorption properties. Owing to the differences in the absorption spectra of oxy- and deoxyhemoglobin, multi-wavelength imaging methods can assess the $\mathrm{SO}_{2}$ in retinal vessels ${ }^{9}$. Currently available optical imaging modalities, such as fundus photography ${ }^{10}$, scanning laser ophthalmoscope (SLO) ${ }^{11}$, and optical coherence tomography (OCT) ${ }^{12-14}$ are scattering based techniques and rely on the back-scattered light from the tissue to form an image. Despite the great progress in these optical imaging techniques, however, they use indirect methods to measure optical absorption. Therefore, the accuracy of these methods is affected by factors such as variation in vessel size ${ }^{15}$, pigmentation ${ }^{16}$, multiple light paths $^{17}$, and vessel wall thickness ${ }^{9}$. For example, in larger vessels the amount of detected backscattered light is much greater than in small vessels ${ }^{17}$, hence the calculation of the optical density and $\mathrm{SO}_{2}$ values can be affected in clinical trials ${ }^{18,19}$. Additionally, it is shown that $\mathrm{SO}_{2}$ variations, induce changes in vessel diameter ${ }^{9}$, which may further alter the amount of backscattered light from the vessel and consequently the $\mathrm{SO}_{2}$ measurements.

Photoacoustic microscopy (PAM), has the unique capability to map the direct optical absorption properties with high resolution in biological tissues ${ }^{20}$. The modality has the potential to overcome the limitations of current ocular imaging methods in functional studies ${ }^{21}$. It has been shown in simulation studies that by increasing the vessel diameter and melanin concentration, the relative error of $\mathrm{SO}_{2}$ 
measurement in the scattering-based method increases. However, the $\mathrm{SO}_{2}$ measurement was insensitive to these parameters in PAM ${ }^{18}$. As these results suggest, PAM can be potentially a more accurate tool in quantifying retinal $\mathrm{SO}_{2}$. However, the disadvantage of PAM in ophthalmic imaging arises from its need to be in contact with the ocular tissue ${ }^{22}$. This physical contact may increase the risk of infection and may cause patient discomfort. Additionally, it applies pressure to the eye and introduces barriers to oxygen diffusion and alter physiological and pathophysiological balance of ocular vasculature function ${ }^{8}$. Additionally, the use of ultrasonic signal detection scheme poses challenges in combining the technology with other imaging modalities ${ }^{22}$.

Recently our group at PhotoMedicine Labs for the first time demonstrated non-contact photoacoustic imaging of the ocular tissue in murine eye using photoacoustic remote sensing (PARS) microscopy ${ }^{23}$. Later we combined the technology with OCT and applied it for in-vivo non-contact imaging of the anterior segment in the mouse eye ${ }^{8}$. We showed the potential of the system for estimation oxygen saturation in the iris vasculature and visualizing melanin content in the RPE. Building on the proven strength of PARS imaging, we further developed the technology to address the aforementioned need in retinal oximetry. In this paper we report our latest progress on in-vivo retinal imaging and present the capability of the reported non-contact multimodal imaging system for visualizing structural and functional information of the rodent retina. To our knowledge, this is the first time a non-contact photoacoustic imaging technique is applied for measuring oxygen saturation in the living retina. This reported work has the potential to advance the diagnosis and treatment of major retinal disorders.

\section{Method}

\subsection{System architecture}

Figure demonstrates the experimental setup of the multimodal PARS-OCT system. Briefly, the output beam of a 532-nm, ytterbium-doped laser (IPG Photonics) with 1.5 ns pulse-width is coupled to a single mode optical fiber. At the output end of the fiber multiwavelength spectral peaks are generated through 
stimulated Raman scattering (SRS). SRS efficiency could be affected by temperature and airflow changes, and random errors and drift in pulse energy ${ }^{24}$. To improve the stability of the SRS peaks, the fiber was kept in a temperature-controlled unit to isolate the airflow and the temperature ${ }^{25}$. The laser output was collimated into $2.1 \mathrm{~mm}$ diameter, then merged with the PARS probe beam centered at 830 (SLD830S-A20, Thorlabs) and the light of SS-OCT (center wavelength: $1060 \mathrm{~nm} ; 100 \mathrm{~nm}$ spectral bandwidth, $60 \mathrm{kHz}$ swept rate, Thorlabs). A two-dimensional galvanometer scanned the combined light beams (GVS012/M, Thorlabs) and relayed them to the eye through a telescopic lens (50:30 ratio) system. The pivot point of the telecentric pair is properly positioned to relay the galvanometer mirrors onto the entrance pupil of the eye, thereby minimizing vignetting. In PARS, pressure waves induced by light absorption were detected by the probe beam. The back-reflected light from the retina is directed towards the detection path. The quarter wave-plate transforms the reflected circularly polarized light back to linearly polarized light, which enables the polarized beam splitter to direct the back-reflected light towards the photodiode. The photodiode outputs are connected to a high-speed digitizer (CSE1442, Gage Applied, Lockport, IL, USA) that performs analog to digital signal conversion. In SS-OCT, backscattered light from the retina first interfered with the reference arm beam, then detected by the built-in dual balanced photodetector. The OCT signal was digitized by a high-speed A/D card (ATS9351, Alazar Technologies Inc., Pointe-Claire, QC, Canada). The raw OCT data was transmitted to a host computer through a PCI-Express interface. OCT system control was implemented in MATLAB platform to automatically control all the operations including system calibration, galvo-scanning, system synchronization, real-time imaging preview and data acquisition. The lateral resolutions of both PARS and SS-OCT were around $\sim 6 \mu \mathrm{m}$ in the retina with a slight difference caused by the different spectral ranges. The axial resolutions of PARS and OCT were $\sim 40 \mu \mathrm{m}$ and $\sim 7.3 \mu \mathrm{m}$, respectively. 


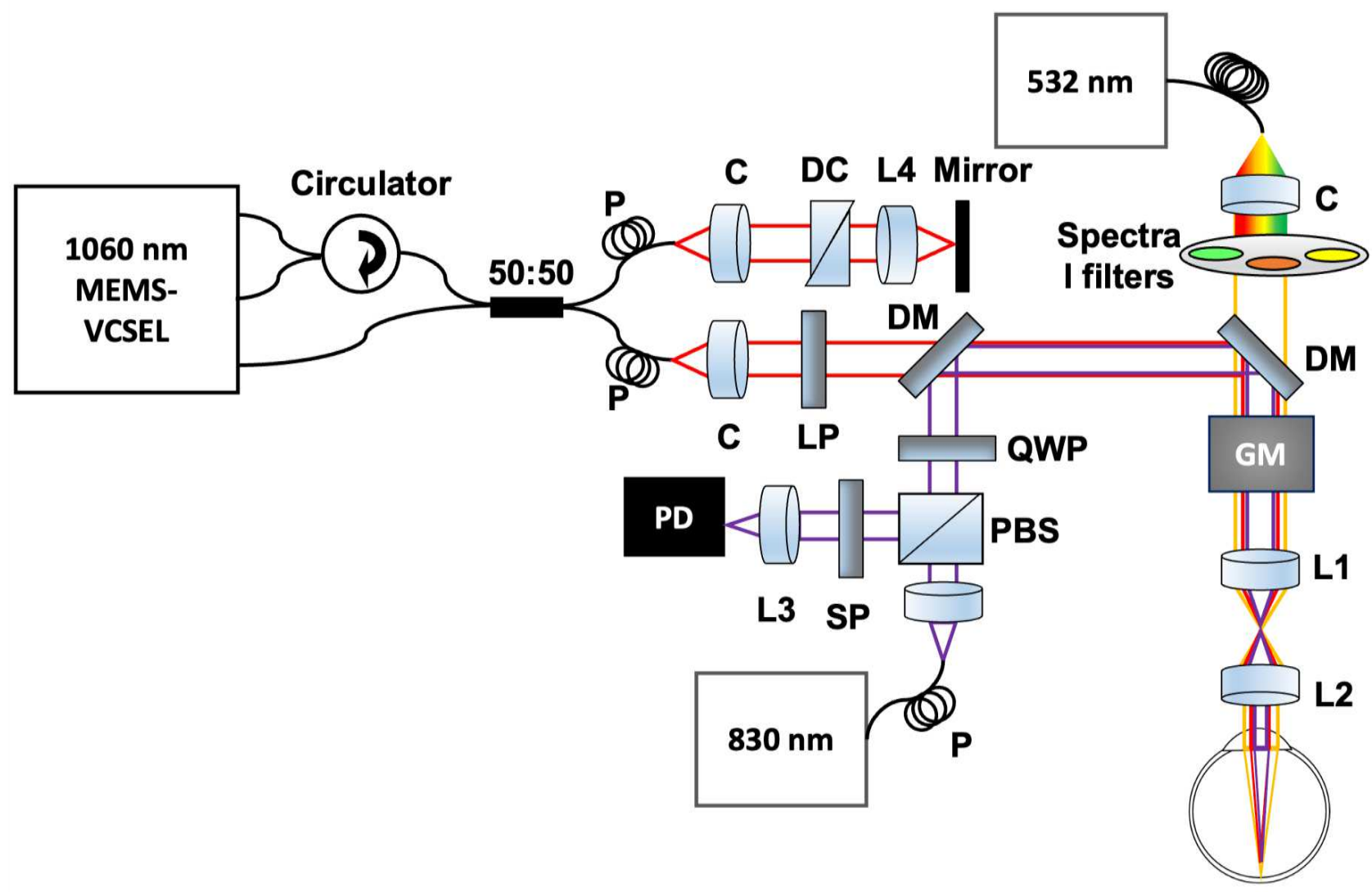

Figure 1. Simplified schematic of the multimodal PARS-OCT system. DM: Dichroic mirror, QWP: Quarter wave plate, PBS: Polarized beam splitter, LP: Long pass filter, GM: Galvanometer mirrors, L: Lens, C: Collimator, PD: Photodiode. DC: Dispersion compensation, P: Polarization controller.

\subsection{Image reconstruction}

All the PARS images shown in this manuscript were formed using a maximum amplitude projection (MAP) of each A-scan for each pixel of the en-face image. The images were produced by direct plotting from interpolated raw data using a Delaunay triangulation interpolation algorithm ${ }^{26}$. All images and signal processing steps were performed in the MATLAB environment.

For each OCT data set, 500 A-lines were acquired for each B-scan. For each A-line trigger, 2448 sampling points were acquired to cover the resultant spectral interferogram, providing a depth ranging distance of $\sim 12 \mathrm{~mm}$. To extract the OCT complex data, reference spectrum was subtracted from the interference signal to remove DC bias, then Fourier transform was performed to extract the depthresolved OCT signal. Images were generated from the raw OCT data and numerically dispersion 
compensated up to the 5 th order with a custom MATLAB algorithm ${ }^{27}$. No additional image postprocessing was used for the OCT images presented in this paper. The volumetric and en-face images were generated from the $3 \mathrm{D}$ data sets with Image ${ }^{28}$.

\subsection{Animal preparation}

All the experimental procedures were carried out in conformity with the laboratory animal protocol approved by the Research Ethics Committee at the University of Waterloo and adhered to the ARVO statement for use of animals in ophthalmic and vision research. All sections of this report adhere to the ARRIVE Guidelines for reporting animal research. Albino rats (NU/NU, Charles River, MA, USA) were imaged to demonstrate the in-vivo capabilities of the system. A custom-made animal holder was used to restrain the animal. The base of the animal holder was lined with a thermal pad to keep the animal body temperature between $36^{\circ}$ and $38^{\circ} \mathrm{C}$. One drop $0.5 \%$ proparacaine hydrochloride (topical anesthetic; Alcaine, Alcon, Mississauga, ON, Canada) was applied to the eye, followed by one drop of $0.5 \%$ tropicamide (pupillary dilator; Alcon). Artificial tears were used frequently ( every 2 minutes) to keep the cornea hydrated. Vital signs, such as respiration rates, heart rates and body temperature were monitored during the experiment.

\section{Results and discussion}

\subsection{Phantom imaging}

Initial calibration of the PARS sub-system was achieved through imaging phantom eye models. First, we used a realistic human eye model to test the feasibility of PARS on the retina. The phantom is shown in Figure 1a, and it was modified from a commercial product (OEM-7, Ocular Instruments, Bellevue, WA). The model consists of the cornea, crystalline lens, aqueous humor, vitreous humor, and artificial retina. Strings of $7 \mu m$ carbon fibers (CF) were placed at the bottom of the model where the artificial retina is located (Figure 1b), and they were imaged using the PARS system. Figure 1c shows representative PARS image acquired from $\mathrm{CF}$ at the back of the human eye model and Figure 1d shows corresponding image 
acquired using PARS scattering mechanism described in our previous report ${ }^{8}$. The PARS excitation and PARS detection are co-aligned, so that the same fibers are in focus in both absorption and scattering contrast images (white arrow).

Then a custom rat eye model was developed to help with the alignment of PARS excitation and detection beam for in-vivo trials. The eye model consisted of a single achromatic lens (63-817; Edmund Optics, Inc) with numerical aperture and curvature close to that of in the rat eye, and a custom 3D printed plastic chamber. Strings of $7 \mu \mathrm{m}$ carbon fibers were located at a fix distance from the lens (Figure 1e) corresponding to the focus of the lens and was approximately equal to the path length of a rat eye ${ }^{29}$. Representative images acquired using absorption and scattering contrast of PARS system are shown in Figure 1f-g. Similar to the human eye model, the excitation and detection beams were co-aligned so that the same CF are in focus in both images (white arrows).

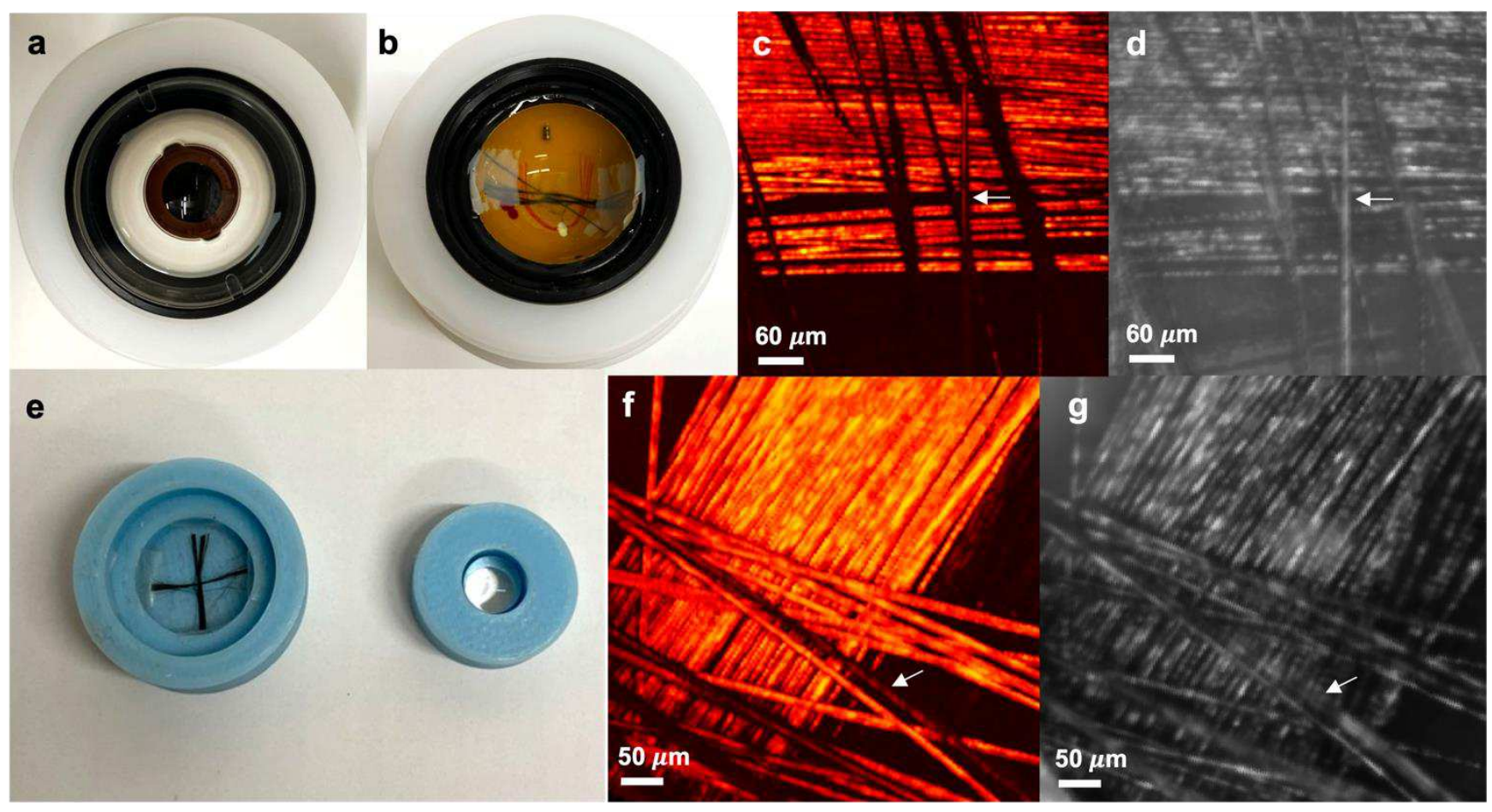

Figure 1. Imaging phantom eye models for human and rat. uman eye model consisting of the cornea, crystalline lens, aqueous humor, vitreous humor, and artificial retina (a). Strings of $7 \mu \mathrm{m}$ carbon fibers are placed at the bottom of the eye model (b). CF image acquired with PARS absorption mechanism (c). CF image acquired using PARS scattering mechanism (d). Custom rat eye model consisting of a single achromatic lens and a 3D printed plastic chamber and strings of carbon fibers (e). PARS absorption and 
scattering contrast images, respectively (f-g). PARS excitation and detection beams are co-aligned so that the same $\mathrm{CF}$ are in focus in both images (white arrows).

\section{2. $\mathrm{SO}_{2}$ accuracy}

The accuracy of the multiwavelength PARS sub-system in measuring oxygen saturation, was investigated by performing in-vitro phantom experiments using freshly collected bovine blood with Sodium Citrated anticoagulant solution. Figure 2 a shows the experimental setup. The setup included a blood reservoir, tubing, oxygen tank, oxygen meter access points and a syringe pump (NE-4000, New Era Pump Systems, Inc.). Different levels of oxygen were delivered to the blood reservoir. Blood samples were drawn before and after image acquisition for $\mathrm{CO}$-oximetry measurements. A clinical grade $\mathrm{CO}$ oximeter (Avoximeter 4000, Instrumentation Laboratory LTD, Richmond Hill, Canada) served as a reference device for measuring blood oxygen saturation.

Images of glass capillary with flowing blood, are acquired at $532 \mathrm{~nm}$ and $558 \mathrm{~nm}$ excitation wavelength (Figure 2b-c). To estimate the $\mathrm{SO}_{2}$, representative signal values were extracted from the two images. It is assumed that the $\mathrm{SO}_{2}$ is homogeneous within the capillary and the mean values are extracted. Compared to the per-pixel basis estimation, the mean value method offers higher accuracy by reducing the impact of random errors. Here, rather than averaging the signal intensity over the entire image, only a sub-region where the target is adequately in-focus is used. The PARS signal intensity within the selected region is averaged to arrive at a representative value associated with each excitation wavelength. The relative concentrations of oxy- and deoxyhemoglobin and the corresponding $\mathrm{SO}_{2}$ value is then calculated. The PARS measurements showed $40 \%$ and $60 \%$ oxygenation which were found to be within $6 \%$ accuracy with the oximeter results. The value is in the range reported by other photoacoustic imaging studies ${ }^{30,31}$, and approaches the ISO-defined acceptable performance criterion for oximeters $(<4 \%)^{32}$. To further improve the accuracy several factors can be considered in future works. For example, nonlinear methods employing more than two optical wavelengths can be implemented to compensate for the absorption saturation effect ${ }^{33}$. Furthermore, to reduce the effect of laser pulse energy fluctuations, a 
more stable tunable source can be employed and ultrashort switching time (a few hundred nanoseconds) among wavelengths can be applied.

a
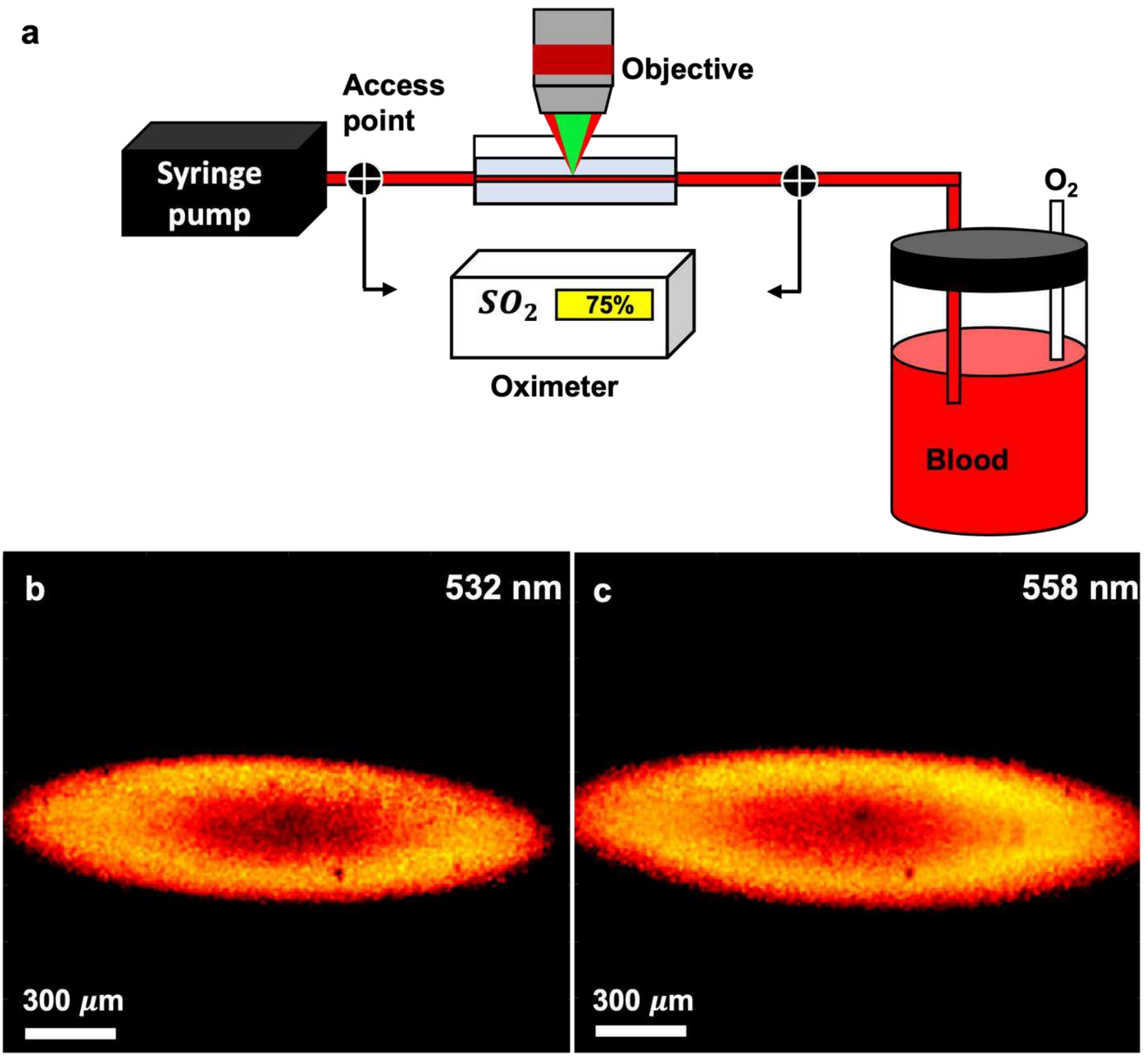

Figure 2. In-vitro bovine blood phantom experiment. Experimental setup of the experiment (a). Images of glass capillary with flowing blood acquired at $532 \mathrm{~nm}$ and $558 \mathrm{~nm}$ excitation wavelengths, respectively $(b-c)$.

\subsection{Retina imaging}

First the capability of the SS-OCT system was tested on the rat retina, and it was used to guide the PARS imaging system. Cross-sectional and volumetric OCT images of the rat retina are demonstrated in Figure

3. Each data set is acquired in $\sim 10$ seconds. Cross-sectional images enable visualization of major retinal 
layers. Retinal nerve fiber layer (NFL) and the retinal ganglion cell layer (GCL) form the innermost (top), highly reflective band. Beneath this are the inner plexiform (IPL), inner nuclear (INL), outer plexiform (OPL), and outer nuclear layer (ONL). In principle, those layers formed of nerve fibers, i.e., IPL and OPL, show high-backscattering, whereas the nuclear layers have low-backscattering. The junction between the inner segment and outer segment of the photoreceptors (I/OS PR) could be visualized, as well as the highly reflective band which comprises the RPE (Figure $3 b$ ). In the OCT images central retinal artery (CRA) remnant were also visible. (Figure 3a). OCT fundus views are also generated by axially summing the merged OCT data set (Figure 3c-e). From the OCT fundus image, as shown in Figure 3 (c), specific anatomy of the rat retina is visualized, including the optic nerve head, some large retinal vessels and optic nerve fiber bundles as indicated by the yellow arrows. Figure $3 \mathrm{e}$ show the retinal microvasculature in the deeper retinal layer (Red arrows).

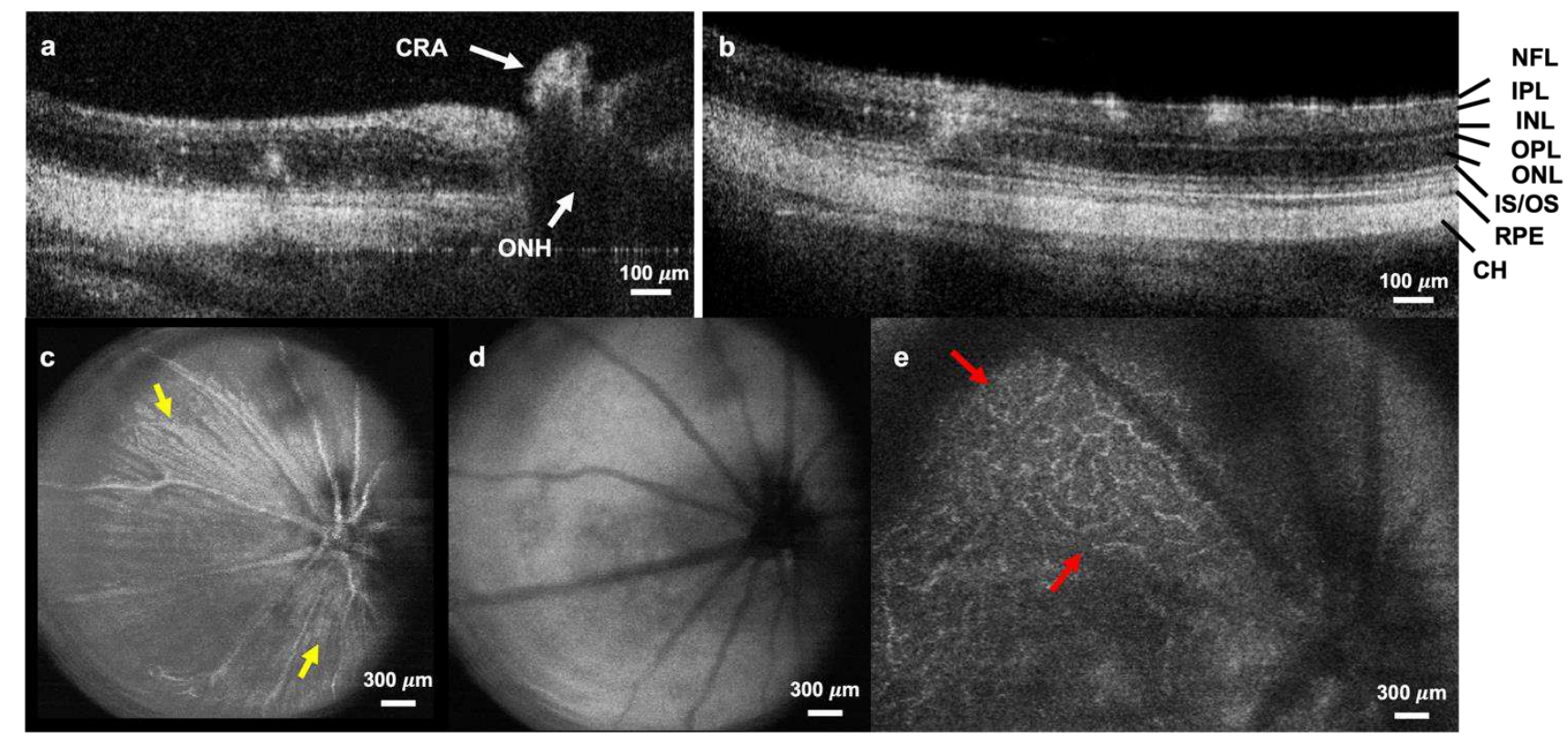

Figure 3. Volumetric and cross-sectional OCT images. cross-sectional images acquired in-vivo from rat retina showing distinct layers of the retina. NFL: nerve fiber layer, IPL: inner plexiform layer, INL: inner nuclear layer, OPL: outer plexiform layer, ONL: Outer nuclear layer, IS/OS junction of inner segment and outer segment layer. RPE retinal pigment epithelium layer, $\mathrm{CH}$ : choroid, CRA: central retinal artery, ONH: optic nerve head (a-b). OCT fundus images visualizing optic nerve head, large retinal vessels, optic nerve fiber bundle (yellow arrows), deeper retinal layer microvasculature (red arrows) (c-e). 
These high-definition structural information of the SS-OCT subsystem can be well complemented by absorption based functional information obtained from PARS microscopy. The axial resolution of the PARS system $(\sim 40 \mu \mathrm{m})$, enables separating the signals of retinal blood vasculature from the RPE melanin given the retina thickness of $\sim 200 \mu \mathrm{m}^{17}$. As a result, PARS signals generated from major vessels can be considered to be mainly from hemoglobin. Figure 4 a demonstrates fundus PARS image acquired from large vessels around $\mathrm{ONH}$ from a $2.6 \mathrm{~mm} \times 2.6 \mathrm{~mm}$ area. Smaller vessels are also visible in the image; however, they are slightly distorted by motion artifacts. Figure $4 \mathrm{~b}$ shows a zoomed-in section of one of the vessels acquired from a similar area where the smaller vasculature is more visible. In the previous report of PARS-OCT system ${ }^{8}$, the PARS scattering contrast provided through the PARS detection beam was introduced. In retinal imaging applications, this capability allows the PARS microscope to be used the same way as fundus photography to image the interior surface of the eye. Figure 4c, show a representative image acquired from the $\mathrm{ONH}$ using scattering contrast of the PARS system. Since $830 \mathrm{~nm}$ beam used in the proposed architecture, has different absorption coefficient for oxygenated and de-oxygenated hemoglobin (higher absorption for oxygenated hemoglobin); arteries appear dark (red arrows), and veins are light (blue arrows) in the image. Therefore, the scattering contrast of PARS microscope, can be used similar to retinal oximeters to measure oxygen saturation in the eye ${ }^{34}$. Relying on the proven strength of the PARS sub-system in providing functional information, the amplitude of multiwavelength PARS signals were employed to estimate $\mathrm{SO}_{2}$ in every vessel (Figure 4d). The calculation is done based on the molecular extinction coefficients of $\mathrm{HbO}_{2}$ and $\mathrm{Hb}$ at $532 \mathrm{~nm}$ and $558 \mathrm{~nm}$ optical wavelengths. The rodent's venous $\mathrm{SO}_{2}$ measured with PARS was around $70 \%$ which agrees with previous reports ${ }^{35}$. Despite the good match between measured $\mathrm{SO}_{2}$ by PARS and the preset $\mathrm{SO}_{2}$ for blood experiments, the in-vivo rodent experiments conditions are different from those in in vitro experiments. For example, there are eye movements during imaging, notable chromatic aberration from the eyeball, etc. Whether such differences will affect the $\mathrm{SO}_{2}$ quantification or not require future 
validation ${ }^{36,37}$. Additionally, it is reported that visual stimulus might increase the retinal vessel diameter and blood flow, as well as affect the retinal $\mathrm{SO}_{2}$ due to retinal neurovascular coupling ${ }^{38}$. These factors may affect the accuracy of the $\mathrm{SO}_{2}$ measurements, and they need further investigation in the future. To our knowledge, this is the first report of optical absorption based retinal $\mathrm{SO}_{2}$ demonstrated in the fundus view. It is important to note that PARS can provide two independent absorption contrast effect. First, is the absorption contrast provided by the excitation laser. In this case, an isosbestic detection laser wavelength can be used to only visualize the excitation effect. Finally, PARS detection laser can also act as the secondary absorption contrast (Fig 5 c.). The combination of these two effects, which is unique to PARS microscopy, can lead to more accurate measurements compared to current methods. This will be investigated further in the future. 


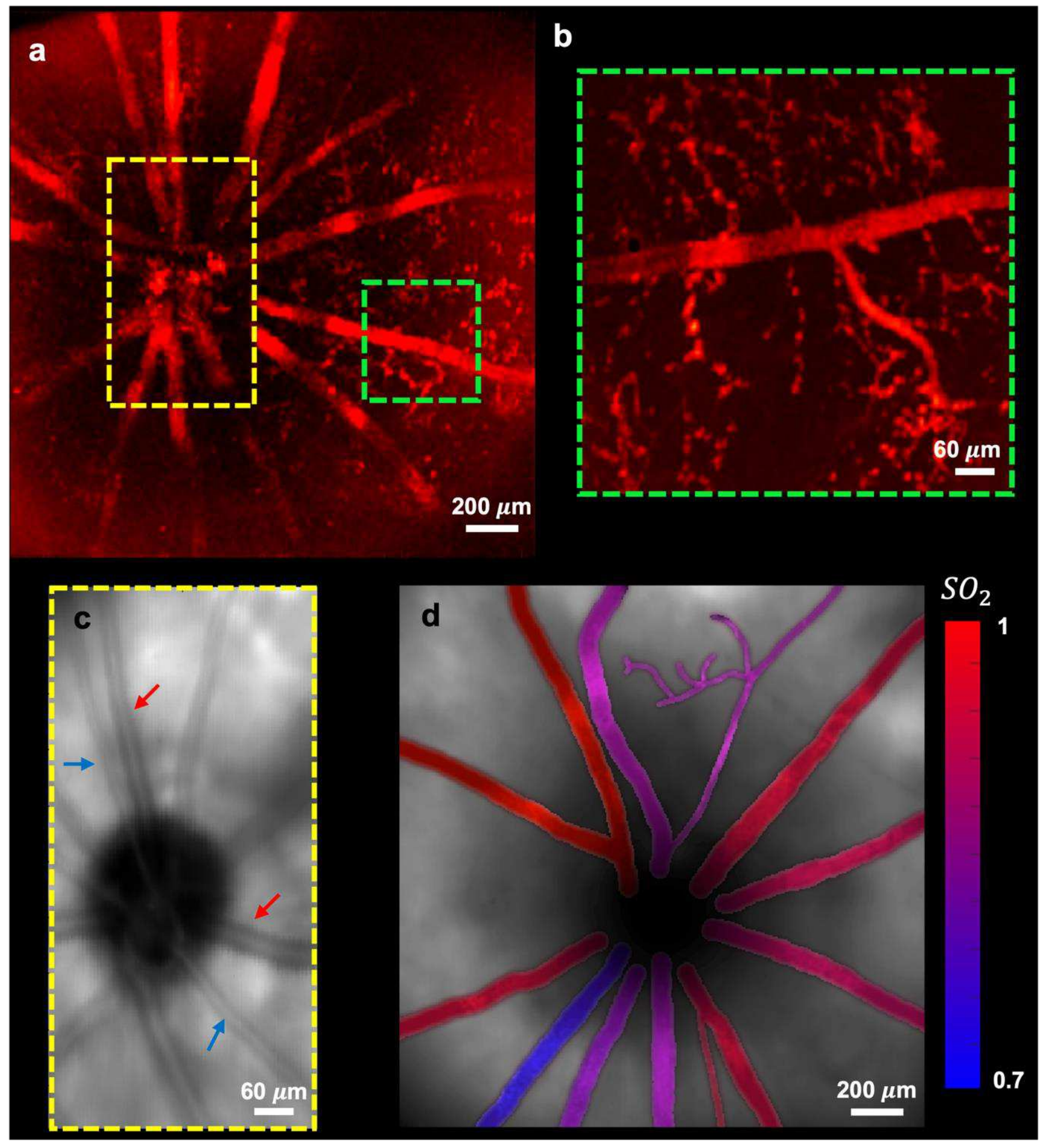

Figure 4. PARS retinal imaging. Fundus PARS image acquired from large vessels around ONH from a $2.6 \mathrm{~mm} \times 2.6 \mathrm{~mm}$ area (a). zoomed-in section of one of the vessels acquired from a similar area with smaller vasculature (b). Fundus image acquired using scattering contrast of PARS system showing arteries (red arrows) and veins (blue arrows) (c). Oxygen saturation map in the retina obtained using multiwavelength PARS imaging (d).

3.4. Ocular light Safety 
Using ANSI standards, the ocular light safety for the developed multimodal PARS-OCT system can be calculated $^{39,40}$. First, the safety of PARS excitation laser is tested using the three ANSI rules: single pulse limit, average power limit, and multiple pulse limit. The pulse duration for the IPG laser used in this study is $t_{1}=1.5 \mathrm{~ns}$. In the imaging setup, assuming that the collimated laser beam after being focused by the eye has a maximum diameter of $20 \mu m$ on the retina, the angular subtense of the source:

$$
\alpha=\frac{20 \mu m}{17 \mathrm{~mm}}=1.2 \mathrm{mrad}<\alpha_{\min }=1.5 \mathrm{mrad}
$$

Where $17 \mathrm{~mm}$ is the focal length of human eye. Therefore, the light source can be considered as a point source.

Rule 1 Since the pulse duration of the laser is $1.5 \mathrm{~ns}$ only the thermal effect needs to be considered and the maximum permissible exposure (MPE) will be:

$$
M P E_{S P}=5 \times 10^{-7} \mathrm{~J} / \mathrm{cm}^{2}
$$

Rule 2 Since the exposure time $(\sim 10 s)$ is longer than the $0.7 s$ and the wavelength is between 400 and $600 \mathrm{~nm}$, dual limits due to both thermal and photochemical effects apply here. For the photochemical effect:

$$
M P E_{p h}=4.36 \times 10^{-2} \mathrm{~J} / \mathrm{cm}^{2}
$$

For the thermal effect:

$$
M P E_{t h}=1.01 \times 10^{-2} \mathrm{~J} / \mathrm{cm}^{2}
$$

Rule 3 tests whether an exposure by a long pulse of duration $n t_{1}$ is safe. Within a laser spot of $20 \mu m$, there are at most two overlapping laser pulses $(n=2)$.

$$
M P E_{r p}=n^{-0.25} \times M P E_{s p}=0.84 \times 5 \times 10^{-7}=4.2 \times 10^{-7} \mathrm{~J} / \mathrm{cm}^{2}
$$


Rule 3 is the most conservative of the three. Considering a pupil diameter of $D=0.7 \mathrm{~cm}$ MPE for a single pulse would be equal to $M P E_{r P} \times\left(\frac{D}{2}\right)^{2} \times \pi \approx 160 \mathrm{~nJ}$. The acquired value is in correspondence to the values reported by other groups ${ }^{41,42}$. In this manuscript the energy of a single pulse is $<$ $150 \mathrm{~nJ}$ which is below the allowed pulse exposure limit.

For $\lambda=380-1400 \mathrm{~nm}$, a spectrally flat limit was recently introduced that recommends MP corneal irradiances of $25 \mathrm{t}^{-0.75} \mathrm{~W} / \mathrm{cm}^{2}$ for $t<10 \mathrm{~s},\left(t=10, M P=4.4 \mathrm{~W} / \mathrm{cm}^{2}\right)$, and $4.0 \mathrm{~W} / \mathrm{cm}^{2}$ for $t>10 \mathrm{~s}^{43}$. Based on the proposed limit the OCT light power on the cornea $(\sim 1.5 \mathrm{~mW})$ and the PARS detection power $(\sim 2-3 \mathrm{~mW})$ are well within the ANSI limits.

There are several aspects of the proposed system that can be further refined for future studies. The current PARS-OCT system was developed using visible excitation wavelength for targeting oxygenated and deoxygenated hemoglobin in the blood. However, the laser safety threshold for ocular imaging is stricter in the visible than in the NIR spectral range. Additionally, the retina is sensitive to the visible light which poses challenges to eye fixation during imaging. Therefore, in future studies, the NIR spectral range can be examined as the excitation wavelength to overcome these issues. Additionally, the oxygen saturation measurement accuracy can be improved by using a more stable tunable light source and using nonlinear methods. In future, the scanning pattern of the SS-OCT system can be modified to enable Doppler OCT and OCT angiography and provide blood flow measurement and vasculature map, respectively. This information can be further combined with the non-contact oxygen saturation measurements of PARS to measure metabolic rate of oxygen consumption in the ocular environment in both small and large vessels. The system can be further applied for evaluating the effects of visually evoked stimulus and how they could change this functional information. It can also be used in longitudinal studies on larger animal models like rabbit and monkeys with size of eyeballs closer to that of humans. Different eye disease 
models (such as Glaucoma, and DR) can be developed in these animals and the efficacy of the system be evaluated in diagnosis and monitoring of them.

\section{Conclusion}

In conclusion, for the first time, a non-contact photoacoustic imaging technique was applied for in-vivo measuring of retinal $\mathrm{SO}_{2}$. It was also the first time multimodal in-vivo imaging of retina was demonstrated using multiwavelength PARS-OCT. The detailed structural information of OCT is well complemented with absorption-based functional information of PARS imaging. The proposed method can be a measure step toward non-invasive measurement of metabolic rate of oxygen consumption in retina, and it can further improve the diagnosis and treatment of important eye diseases.

\section{Acknowledgements:}

The authors would like to thank Jean Flanagan for assistance with animal-related procedures. The authors would also like to thank Kevan Bell, Benjamin Ecclestone for their support and help. The authors acknowledge funding from New Frontiers in Research Fund - Exploration (NFRFE-2019-01012); Natural Sciences and Engineering Research Council of Canada (DGECR-2019-00143, RGPIN201906134); Canada Foundation for Innovation (JELF \#38000); Mitacs (IT13594); Centre for Bioengineering and Biotechnology (CBB Seed fund); University of Waterloo and illumiSonics (SRA \#083181).

\section{Additional Information}

\section{Conflict of interest:}

Author P. Haji Reza has financial interests in illumiSonics Inc. IllumiSonics partially supported this work. All other authors have no competing/conflict of interest.

\section{References}


1. Chen, H., Rogalski, M. M. \& Anker, J. N. Advances in functional X-ray imaging techniques and contrast agents. Phys Chem Chem Phys 14, 13469-13486 (2012).

2. Antonetti, D. A., Klein, R. \& Gardner, T. W. Diabetic retinopathy. N Engl J Med 366, 1227-1239 (2012).

3. Wangsa-Wirawan, N. D. \& Linsenmeier, R. A. Retinal oxygen: fundamental and clinical aspects. Arch Ophthalmol 121, 547-557 (2003).

4. Ciulla, Thomas A., Alon Harris, Hak S. Chung, Ronald P. Danis, Larry Kagemann, Lissa McNulty, Linda M. Pratt, and Bruce J. Martin. "Color Doppler imaging discloses reduced ocular blood flow velocities in nonexudative age-related macular degeneration." American journal of ophthalmology 128, no. 1 (1999): 7580.

5. Eliasdottir, T. S. Retinal oximetry and systemic arterial oxygen levels. Acta Ophthalmol 96 Suppl A113, 144 (2018).

6. B. A. Berkowitz and C. A. Wilson, "Quantitative mapping of ocular oxygenation using magnetic resonance imaging," Magn Reson Med, vol. 33, no. 4, pp. 579-581, Apr. 1995, doi: 10.1002/mrm.1910330419..

7. F inikova, Olga S., Artem Y. Lebedev, Alexey Aprelev, Thomas Troxler, Feng Gao, Carmen Garnacho, Silvia Muro, Robin M. Hochstrasser, and Sergei A. Vinogradov. "Oxygen microscopy by two-photonexcited phosphorescence." Chemphyschem: a European journal of chemical physics and physical chemistry 9, no. 12 (2008): 1673.

8. Z. Hosseinaee, Nima Abbasi, N. Pellegrino, L. Khalili, L. Mukhangaliyeva, and P. Haji Reza, "Functional and structural ophthalmic imaging using noncontact multimodal photoacoustic remote sensing microscopy and optical coherence tomography," Sci Rep, vol. 11, no. 1, Art. no. 1, Jun. 2021, doi: 10.1038/s41598-02190776-5.

9. Harris, A., Dinn, R. B., Kagemann, L. \& Rechtman, E. A review of methods for human retinal oximetry. Ophthalmic Surg Lasers Imaging 34, 152-164 (2003).

10. Huang, Z. et al. Retinal choroidal vessel imaging based on multi-wavelength fundus imaging with the guidance of optical coherence tomography. Biomed. Opt. Express, BOE 11, 5212-5224 (2020).

11. Sharp, P. F., Manivannan, A., Xu, H. \& Forrester, J. V. The scanning laser ophthalmoscope-a review of its role in bioscience and medicine. Phys. Med. Biol. 49, 1085-1096 (2004). 
12. Aumann, S., Donner, S., Fischer, J. \& Müller, F. Optical Coherence Tomography (OCT): Principle and Technical Realization. in High Resolution Imaging in Microscopy and Ophthalmology: New Frontiers in Biomedical Optics (ed. Bille, J. F.) 59-85 (Springer International Publishing, 2019). doi:10.1007/978-3-03016638-0_3.

13. Chen, C.-L. \& Wang, R. K. Optical coherence tomography based angiography [Invited]. Biomed. Opt. Express, BOE 8, 1056-1082 (2017).

14. Drexler, W. \& Fujimoto, J. G. Optical coherence tomography: technology and applications. (Springer Science \& Business Media, 2008).

15. Buerk, D. G., Shonat, R. D., Riva, C. E. \& Cranstoun, S. D. O2 gradients and countercurrent exchange in the cat vitreous humor near retinal arterioles and venules. Microvasc Res 45, 134-148 (1993).

16. Hammer, M., Vilser, W., Riemer, T. \& Schweitzer, D. Retinal vessel oximetry-calibration, compensation for vessel diameter and fundus pigmentation, and reproducibility. J Biomed Opt 13, 054015 (2008).

17. Hammer, M., Leistritz, S., Leistritz, L. \& Schweitzer, D. Light paths in retinal vessel oxymetry. IEEE Trans Biomed Eng 48, 592-598 (2001).

18. Liu, W., Jiao, S. \& Zhang, H. F. Accuracy of retinal oximetry: a Monte Carlo investigation. J Biomed Opt 18, 066003 (2013).

19. J iao, Shuliang, Minshan Jiang, Jianming Hu, Amani Fawzi, Qifa Zhou, K. Kirk Shung, Carmen A. Puliafito, and Hao F. Zhang. "Photoacoustic ophthalmoscopy for in vivo retinal imaging." Optics express 18, no. 4 (2010): 3967-3972.

20. Yao, J. \& Wang, L. V. Sensitivity of photoacoustic microscopy. Photoacoustics 2, 87-101 (2014).

21. Z hang, Wei, Yanxiu Li, Yixin Yu, Katherine Derouin, Yu Qin, V. Phuc Nguyen, Xiaobo Xia, Xueding Wang, and Yannis M. Paulus. "Simultaneous photoacoustic microscopy, spectral-domain optical coherence tomography, and fluorescein microscopy multi-modality retinal imaging." Photoacoustics 20 (2020): 100194

22. Song, Wei, Qing Wei, Wenzhong Liu, Tan Liu, Ji Yi, Nader Sheibani, Amani A. Fawzi, Robert A. Linsenmeier, Shuliang Jiao, and Hao F. Zhang. "A combined method to quantify the retinal metabolic rate 
of oxygen using photoacoustic ophthalmoscopy and optical coherence tomography." Scientific reports 4 , no. 1 (2014): 1-7.

23. Z. Hosseinaee, L. Khalili, J. A. T. Simmons, K. Bell, K. Bell, and P. H. Reza, "Label-free, non-contact, in vivo ophthalmic imaging using photoacoustic remote sensing microscopy," Opt. Lett., OL, vol. 45, no. 22, pp. 6254-6257, Nov. 2020, doi: 10.1364/OL.410171.

24. Liu, C., Chen, J., Zhang, Y., Zhu, J. \& Wang, L. Five-wavelength optical-resolution photoacoustic microscopy of blood and lymphatic vessels. AP 3, 016002 (2021).

25. Hosseinaee, Zohreh, Benjamin Ecclestone, Nicholas Pellegrino, Layla Khalili, Lyazzat Mukhangaliyeva, Paul Fieguth, and Parsin Haji Reza. "Functional photoacoustic remote sensing microscopy using a stabilized temperature-regulated stimulated Raman scattering light source." Optics Express 29, no. 19 (2021): 2974529754.

26. Chen, L. \& Xu, J. OPTIMAL DELAUNAY TRIANGULATIONS. Journal of Computational Mathematics 22, 299-308 (2004).

27. Cense, Barry, Nader A. Nassif, Teresa C. Chen, Mark C. Pierce, Seok-Hyun Yun, B. Hyle Park, Brett E. Bouma, Guillermo J. Tearney, and Johannes F. De Boer. "Ultrahigh-resolution high-speed retinal imaging using spectral-domain optical coherence tomography." Optics express 12, no. 11 (2004): 2435-2447.

28. Schneider, C. A., Rasband, W. S. \& Eliceiri, K. W. NIH Image to ImageJ: 25 years of image analysis. Nature Methods 9, 671-675 (2012).

29. Lozano, D. C. \& Twa, M. D. Development of a Rat Schematic Eye From In Vivo Biometry and the Correction of Lateral Magnification in SD-OCT Imaging. Invest. Ophthalmol. Vis. Sci. 54, 6446-6455 (2013).

30. Gehrung, M., Bohndiek, S. E. \& Brunker, J. Development of a blood oxygenation phantom for photoacoustic tomography combined with online pO2 detection and flow spectrometry. JBO 24, 121908 (2019).

31. R. Bulsink, M. Kuniyil Ajith Singh, M. Xavierselvan, S. Mallidi, W. Steenbergen, and K. J. Francis, “Oxygen Saturation Imaging Using LED-Based Photoacoustic System,” Sensors (Basel), vol. 21, no. 1, p. 283, Jan. 2021, doi: 10.3390/s21010283. 
32. ISO 80601-2-61:2017. ISO

33. Liu, C., Liang, Y. \& Wang, L. Optical-resolution photoacoustic microscopy of oxygen saturation with nonlinear compensation. Biomed. Opt. Express 10, 3061 (2019).

34. Beach, J. Pathway to Retinal Oximetry. Transl Vis Sci Technol 3, 2 (2014).

35. Gray, L. H., and J. M. Steadman. "Determination of the oxyhaemoglobin dissociation curves for mouse and rat blood." The Journal of physiology 175, no. 2 (1964): 161-171.

36. Harmening, W. M., Tiruveedhula, P., Roorda, A. \& Sincich, L. C. Measurement and correction of transverse chromatic offsets for multi-wavelength retinal microscopy in the living eye. Biomed Opt Express 3, 20662077 (2012).

37. Remtulla, S. \& Hallett, P. E. A schematic eye for the mouse, and comparisons with the rat. Vision Res $\mathbf{2 5}$, 21-31 (1985).

38. Radhakrishnan, H. \& Srinivasan, V. J. Multiparametric optical coherence tomography imaging of the inner retinal hemodynamic response to visual stimulation. J Biomed Opt 18, 86010 (2013).

39. Delori, F. C., Webb, R. H. \& Sliney, D. H. Maximum permissible exposures for ocular safety (ANSI 2000), with emphasis on ophthalmic devices. J. Opt. Soc. Am. A, JOSAA 24, 1250-1265 (2007).

40. ANSI Z136.1-2014: Safe Use of Lasers - ANSI Blog. The ANSI Blog https://blog.ansi.org/2015/07/ansiz1361-2014-safe-use-of-lasers/ (2015).

41. Tian, C., Zhang, W., Mordovanakis, A., Wang, X. \& Paulus, Y. M. Noninvasive chorioretinal imaging in living rabbits using integrated photoacoustic microscopy and optical coherence tomography. Opt. Express, OE 25, 15947-15955 (2017).

42. Liu, W. \& Zhang, H. F. Photoacoustic imaging of the eye: A mini review. Photoacoustics 4, 112-123 (2016).

43. Sliney, David, Danielle Aron-Rosa, Francois DeLori, Franz Fankhauser, Robert Landry, Martin Mainster, John Marshall et al. "Adjustment of guidelines for exposure of the eye to optical radiation from ocular instruments: statement from a task group of the International Commission on Non-Ionizing Radiation Protection (ICNIRP)." Applied optics 44, no. 11 (2005): 2162-2176 


\section{Author Contributions:}

Z.H. designed and constructed the PARS-OCT system, conducted the experiments, wrote the main manuscript, and prepared the figures. N.P. developed and implemented the signal unmixing method. N.A. constructed the software of the OCT system and helped with the animal imaging experiments. T.A. helped with the $\mathrm{SO}_{2}$ accuracy study. J.A.T.S. helped with the design of the rat retina phantom. P.F. contributed to the interpretation of the results and provide guidance. P.H.R. conceived the project and acted as the primary investigator. All the authors reviewed the manuscript. 